\title{
Adjustable gravitational valves. From the conception in 1996 to first implantations 2008
}

\author{
Alfred Aschoff*, Michael Kiefer, Uwe Kehler, Bahram Hashemi and \\ Andreas Unterberg
}

\author{
Address: University of Heidelberg, Department of Neurosurgery. Im Neuenheimer Feld 400, 69120 Heidelberg, Germany \\ Email: Alfred Aschoff* - Alfred_Aschoff@med.uni-heidelberg.de \\ * Corresponding author
}

from 53rd Annual Meeting of the Society for Research into Hydrocephalus and Spina Bifida Belfast, UK. 24-27 June 2009

Published: 27 November 2009

Cerebrospinal Fluid Research 2009, 6(Suppl 2):S22 doi:I0.1 186/1743-8454-6-S2-S22

This abstract is available from: http://www.cerebrospinalfluidresearch.com/content/6/S2/S22

(c) 2009 Aschoff et al; licensee BioMed Central Ltd.

\section{Background}

In 1975 Hakim conceived and Schulte constructed the first gravitational (g)-valve, which used implemented balls for a position-dependent automatic adjustment of the valve-resistance, in order to compensate for the hydrostatic over-drainage in the upright position. In spite of excellent tests in vitro the Hakim-Lumbar was rarely used and had no commercial success, probably due to an exclusive design for lumboperitoneal shunts. In addition the obligate vertical orientation was difficult to achieve because of the round valve body. In the early 90's the forgotten concept was rediscovered simultaneously by Richard/Block, Affeld/Miethke, Aschoff, Sophysa and Chhabra and let to numerous new g-valves with a superior handling.

In 1991 the combination of g-with adjustable valves was proposed and realized in 1993 (Aschoff). Since 1994 they were routinely implanted by an increasing number of users. Actually about 40 g-valve-studies show reduced quotes of clinical relevant over-drainage; subdurals requiring evacuations were $20-30 \%$ only compared to conventional valves. However an inappropriate selection of pressure ranges with consecutive g-valve revisions remained a problem. In 1996 the author suggested adjustable g-valves and developed 2000/1 seven detailed technical solutions. The idea was picked up by Miethke, who patented the first ProSA in 2004 und an improved version, which passed the CE-tests in 2008.

\section{Materials and methods}

The ProSA implies an excenter fixed on a magnetic rotor, which varies the tension of a spring counteracting the weight of a gravitational ball. The opening pressure in vertical can be changed stepless between 0-40 $\mathrm{cmH}_{2} \mathrm{O}$. A brake excludes unintentional readjustments by magnets and MRI up to $3 \mathrm{~T}$. The implantation is possible on the sternum or lateral head. Like all g-valves, a strict vertical orientation to the longitudinal body axis is essential. The ProSA can be combined with any simple DP- or adjustable valves.

\section{Results}

From Dec 2008 until now 15 implantations were clinically successful (actualized data follow in June). Three of them with decompensating complicated hydrocephalus had a perioperative ICP-measurement; all showed physiological ICPs in upright and horizontal.

\section{Conclusion}

Adjustable g-valves are a consequent further development of shunt technology and verifiable effective in vivo. A prospective multicenter trial has been launched (Kehler/ Kiefer 08). 